\title{
Dielectric function for the Anderson model
}

\author{
A RAMAKANTH \\ Department of Physics, Kakatiya University, Warangal 506009, India
}

\begin{abstract}
In recent years the study of alloys and compounds containing rare-earth and actinide elements is receiving increasing attention. The Anderson model is most popularly used for studying the theory of these systems. As it displays a large number of anomalous characters in magnetic and electrical properties, it was [elt worthwhile to study the dielectric properties of this model. Using the linear response theory of Kubo, the energy and wave wector-dependent dielectric lunction $\bar{c}(\bar{q}, E)$ is related to the retarded Oreen's function of Fourier components of electron density nuctuations $\left\langle\rho_{\bar{a}} ; p_{-\bar{q}}\right\rangle$. Thus a many-body calculation of $\varepsilon(\bar{q}, E)$ requires the calculation of $\left\langle\rho_{\bar{q}} ; \rho_{-\bar{q}}\right\rangle$. The Greens function is calculated using the equation-of-motion method with RPA decoupling. Further, since certain ensemble averages are required as inpuls to the calculation, the relevant single-particle Green's functions are also cvaluated.
\end{abstract}

Keywords. Anderson model; dielectric function; linear response.

\section{Introduction}

The frequency and wave vector-dependent dielectric function ts one of the important response functions. The study of diclectric function yields information not only about the dielectric properties of the system but also the collective modes of excitation in the many-body system. The poles of the dielectric function give the plasmon frequency. The wave vector dependence of the plasmon frequency gives insights into the interactions in the system. The dielectric function determines the charge induced in an otherwise electrically neutral system when an external charge is introduced. If it is assumed that the response of the system to an external perturbation depends linearly on the perturbation, the results of linear response theory of Kubo (1957) can be used. Within Kubo's formalism, the dielectric function of an interacting electron system can be expressed in terms of a retarded Green's function. Once this is done, the problem of evaluation of the dielectric function reduces to that of calculating the Green's function. Standard techniques are now available in many-body theory to evaluate the Green's functions (Mahan 1981).

In the recent past, attention of theorists and experimentalists was focussed on alloys and compounds containing rare-earth and actinide group of elements (Newns and Read 1987). This is because the systems show a rich variety of phenomena and display anomalous behaviour in many physical properties. They display insulator-tometal transition as in the case of well-known black-to-gold transition of SmS (Jayaraman 1974). The phenomenon of mixed valence was observed in these systems (Varma 1976). The concept of heavy Fermions was introduced while investigating these systems (Lee et al 1986). Some of these compounds also show heavy Fermion superconductivity which is suspected to be basically different from the conventional BCS-superconductivity. Further, many of these systems show anomalous behaviour in electrical conductivity (Wohlleben and Wittershagen 1985), magnetic susceptibility 
charge $\rho_{\text {lot }}(\bar{v}, i)$. Writing the Maxwell's equations

$$
\begin{aligned}
& \operatorname{div} \bar{D}=\rho_{\mathrm{ex}}(\bar{r}, t) \\
& \operatorname{div} \bar{E}=\frac{1}{\varepsilon_{0}} \rho_{\mathrm{int}}(r, t)=\frac{1}{\varepsilon_{0}}\left(\rho_{\text {ind }}+\rho_{\mathrm{ex}}\right)
\end{aligned}
$$

in the $\langle\bar{q}, E)$ space,

$$
\begin{aligned}
& i \bar{q} \cdot \bar{D}(\bar{q}, E)=\rho_{\mathrm{cx}}(\bar{q}, E) \\
& i \bar{q} \cdot \overline{\mathscr{E}}(\bar{q}, E)=\frac{1}{\varepsilon_{0}}\left[\rho_{\mathrm{ex}}(\bar{q}, E)+\rho_{\mathrm{ind}} \cdot(\bar{q}, E)\right]
\end{aligned}
$$

and using the material equation

$$
\bar{D}(\bar{q}, E)=\varepsilon_{0} \varepsilon(\bar{q}, E) \overline{\mathscr{E}}(\bar{q} . E)
$$

where $\varepsilon(\bar{q}, E)$ is the dielectric function. We get the relation

$$
\rho_{\text {lind }}(\bar{q}, E)=\left(\frac{1}{\varepsilon(\bar{q}, E)}-1\right) \rho_{\mathrm{ex}}(\bar{q}, E) \text {. }
$$

When an external charge is introduced into the system, it interacts with the existing elcctron charge distribution - $e p(\vec{r})$ where $\rho(\vec{r})$ is the density distribution function. Expressing the two charge distributions in terms of the Fourier components

$$
\begin{aligned}
& \rho_{\mathrm{ex}}(\bar{r}, t)=\frac{1}{2 \pi \hbar \hat{h}} \int_{-\infty}^{\infty} \mathrm{d} E \sum_{\bar{q}} \rho_{\mathrm{ex}}(\bar{q}, E) \exp (i \bar{q} \cdot \bar{r}) \exp \left[(-i / h)\left(E+i 0^{+}\right) t\right] \\
& -e \rho(\bar{r})=\frac{-e}{\Omega} \sum_{\bar{q}} \rho_{\bar{q}} \exp (i \bar{q} \cdot \bar{r})
\end{aligned}
$$

where $\Omega$ is the volume of the system, the interaction energy between the two charge distributions

$$
V=\frac{-e}{4 \pi \varepsilon_{\mathrm{n}}} \iint \mathrm{d} \bar{r} \mathrm{~d} \vec{r}^{\prime} \frac{\rho_{e x}(\bar{r}, t) \rho\left(\bar{r}^{\prime}\right)}{\left|\vec{r}-\vec{r}^{\prime}\right|}
$$

can be written as

where

$$
V=\frac{-1}{2 \pi \hbar \Lambda} \int_{-\infty}^{\infty} \mathrm{d} E \exp \left[(-i / \hbar)\left(E+i 0^{+}\right) t\right] \sum_{\tilde{q}} v(\bar{q}) \rho_{-\bar{q}} \rho_{\mathrm{ex}}(\bar{q}, E)
$$

$$
v(\bar{q})=-e / \varepsilon_{0} q^{2}
$$

In deriving (20) use has been made of the result

$$
\iint \mathrm{d} r \mathrm{~d} r^{\prime} \frac{\exp \left[i\left(\bar{q} \cdot \bar{r}+\bar{q}^{\prime} \cdot \bar{r}^{\prime}\right)\right]}{\left|\bar{r}-\bar{r}^{\prime}\right|}=\frac{4 \pi \Omega}{q^{2}} \delta_{\bar{q}_{-}-\bar{q}^{\prime}} .
$$

Defining further

$$
F(\bar{q}, t)=\frac{v(\bar{q})}{2 \pi \bar{h}} \int \mathrm{d} E \exp \left[(-i / h)\left(E+i 0^{+}\right) t\right] \rho_{\mathrm{ex}}(\bar{q}, E)
$$


which can be interpreted as the $\bar{q}$-component of the scalar perturbation field created by the excess charge, we can write (21) as

$$
V=\frac{1}{\Omega} \sum_{\bar{u}} \rho_{\bar{q}} F(\bar{q}, t) .
$$

The response of the system to the introduction of the external charge $\rho_{\mathrm{ex}}(\bar{r}, t)$ is the induced charge $\rho_{\text {ind }}(\vec{r}, t)$. The ensemble average of the $\vec{q}$-component of the induced charge

$$
\left\langle\rho_{\text {ind }}(\bar{q}, t)\right\rangle=-e\left(\left\langle\rho_{\vec{q}}\right\rangle-\left\langle\rho_{\bar{p}}\right\rangle_{0}\right)=-e\left\langle\rho_{\vec{q}}\right\rangle .
$$

In view of (24) and (25), in the Kubo formula (11), $A=\rho_{q}$ and $B=\rho_{-\pi}$. Therefore we can write

$$
\left\langle\rho_{\mathrm{in \dot {a }}}(\bar{q}, t)\right\rangle=\frac{1}{2 \pi \hbar \Omega} \sum_{\bar{q}} \int_{-\infty}^{\infty} \mathrm{d} E F\left(q^{\prime}, E\right) \exp \left[(-i / h)\left(E+i 0^{+}\right) t\right]\left\langle\left\langle\rho_{\bar{q}} ; p_{-\bar{q}}\right\rangle\right.
$$

writing in the energy representation and using the property

We get

$$
\left\langle\rho_{\bar{q}} ; \rho_{-\bar{q}^{\prime}}\right\rangle=\left\langle\left\langle\rho_{\dot{q}} ; \rho_{-\bar{q}}\right\rangle \delta_{\bar{q}, \bar{q}^{*}}\right.
$$

$$
\left\langle\rho_{\text {ind }}(\vec{q}, E)\right\rangle=\frac{v(\vec{q})}{h \Omega} \rho_{c x}(\bar{q}, E)\left\langle\rho_{\vec{q}} ; \rho_{-\vec{q}}\right\rangle \text {. }
$$

Comparing this with (17) we get an expression for the energy and momentum-dependent diclectric function as

$$
\frac{1}{\varepsilon(\bar{q}, \bar{E})}=1+\frac{v(\bar{q})}{h \Omega}\left\langle\left\langle\rho_{\bar{q}} ; \rho_{-\bar{q}}\right\rangle\right.
$$

In the next section we propose to calculate the Green's function $\left.\left\langle\rho_{\bar{q}} ; \rho_{.}\right\rangle\right\rangle$which appears on the right side of (28) for the Anderson model.

\section{Calculation of $\left\langle\left\langle\rho_{q} ; \rho_{-\dot{q}}\right\rangle\right.$ for the Anderson model}

The Hamiltonian for the periodic Anderson model reads as

$$
H=H_{c}+H_{J}+H_{J J}+H_{p},
$$

where $I_{\mathrm{c}}$ represents the conduction electron system and is given by

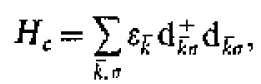

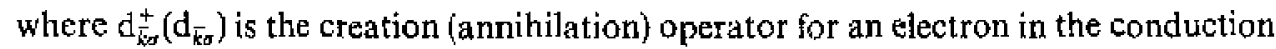
band with momentum $\bar{k}$ and spin $a$. The periodic array of localized $f$-electrons is described by

$$
H_{f}=E_{f} \sum_{i, \sigma} f_{i \sigma}^{+} f_{i, r}
$$


where $E_{f}$ is the energy of the $f$-electron and $f_{i \sigma}^{+}\left(f_{i \sigma}\right)$ is the creation (annihilation) operator for an $f$-electron with spin $\sigma$ at the lattice site $i$. The strong on-site correlation among the f-electrons is assumed to be of the Hubbard type (Hubbard 1963) and is given by

$$
H_{f J}=\frac{U}{2} \sum_{i, \sigma} f_{i \sigma}^{+} f_{i \sigma} f_{l-\sigma}^{+} f_{i-\sigma}
$$

The hybridization interaction between the two electron subsystems, namely, the conduction and localized electrons is given by

$$
H_{u}=\sum_{i, j, \sigma} V_{i j}\left(\mathrm{~d}_{i \sigma}^{+} f_{j \sigma}+\text { h.c. }\right) .
$$

However, we will assume this interaction to be on-site and $V_{i j}$ to be independent of lattice sites so that

$$
H_{\mathrm{v}}=V \sum_{i, \sigma}\left(\mathrm{d}_{i \sigma}^{+} f_{l \sigma}+\text { h.c. }\right) \text {. }
$$

We now employ a similarity transformation (Riseborough and Mills 1980) to express the Anderson Hamiltonian in terms of new operators $\alpha_{\bar{k}, j, \omega^{*}}$. The similarity transformation is defined by

and

$$
\mathrm{d}_{\overline{k, s}}=\sum_{j=1,2} A(\widetilde{k}, j) \alpha_{\bar{k}_{j}, \sigma}
$$

$$
f_{\tilde{R}_{, \sigma}}=\sum_{j=1,2} B\left(\bar{k}_{1} j\right) \alpha_{k_{j, j, \sigma}}
$$

The Hamiltonian in the transiormed representation reads as

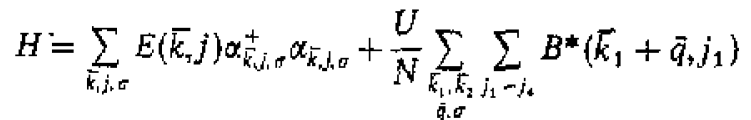

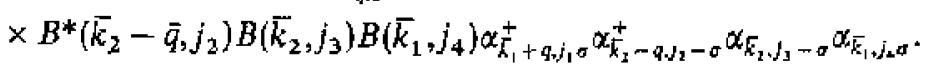

The operators $\alpha_{k, j, \sigma}^{+}\left(\alpha_{\bar{k}, j, \sigma}\right)$ can now be interpreted as the creation (annihilation) operator for an electron in the hybridized band $j$ with momentum $\vec{k}$ and spin $\sigma . E(\bar{k}, j)$ can be interpreted as the energy of the electron in the hybridized band $j$ and is given by

$$
E\left(\bar{k}, \frac{1}{2}\right)=\frac{1}{2}\left[\varepsilon_{\bar{k}}+E_{f} \pm\left(\left(\varepsilon_{\bar{k}}-E_{f}\right)^{2}+4 V^{2}\right)^{1 / 2}\right] .
$$

Expressing $\rho_{\bar{q}}$ in the second quantized representation and transforming to $\alpha$-operators we get

$$
\begin{aligned}
& \rho_{\bar{q}}=\sum_{\bar{k}_{, \sigma} \sigma} \mathrm{d}_{\overline{h_{1}},}^{+} \mathrm{d}_{\bar{k}+q, \sigma} \\
& =\sum_{\substack{k_{1}, \sigma_{1} \\
j_{1}, j_{2}}} A^{*}\left(\bar{k}, j_{1}\right) A\left(\bar{k}+\bar{q}, j_{2}\right) \alpha_{k_{k}, j_{1}, \sigma^{2}}^{+} \alpha_{\bar{k}+\tilde{q}_{j}, j_{2}, \sigma^{*}}
\end{aligned}
$$


The two-particle Green's function that we wish to calculate is then given by

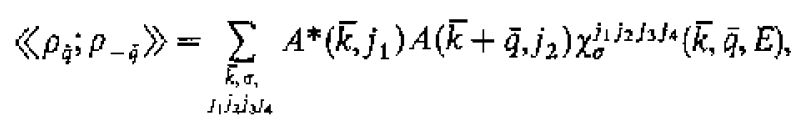

where the reduced susceptibility $\chi_{\sigma}^{j_{1} j_{2} t_{3} / d_{4}}(\bar{k}, \bar{q}, E) \equiv \chi_{a}^{j_{1} / j_{2} j_{\alpha} j_{\alpha}}(\bar{k})$ is given by

with

$$
\chi_{\sigma}^{j_{1} j_{2} j_{3} j_{4}}(\bar{k})=\left\langle\left\langle\alpha_{k, j_{1}, \sigma}^{+} \alpha_{\bar{k}+\bar{q}, j_{2}, \sigma} ; \mathscr{W}_{-q}^{j_{3} j_{A}}\right\rangle\right.
$$

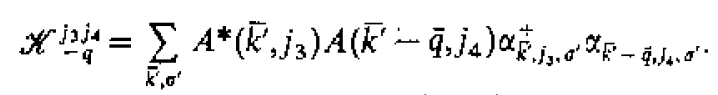

We calculate reduced susceptibility using the equation-of-motion method (Zubarev 1960). This is given by

$$
\begin{aligned}
& E \chi_{\sigma}^{j_{j} j_{2} j_{3} j_{4}}(\bar{k})=\left\langle\left[\alpha_{\bar{k}, j_{1}, \theta}^{+} \alpha_{\bar{k}+\bar{q}, j_{2}, \sigma}, \mathscr{K}_{-4}^{j_{3} j_{4}}\right]\right\rangle
\end{aligned}
$$

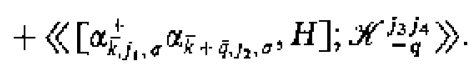

The commutator in the second term on the right side of (42) is calculated using (36) for $H$ to yield

where

$$
\begin{aligned}
& {\left[E-E\left(\bar{k}+q, j_{2}\right)+E\left(\overline{k_{s}} j_{1}\right)\right] \chi_{\sigma}^{j_{1} j_{2} j_{j} j_{4}}(\bar{k})} \\
& =A^{*}\left(\bar{k}+\bar{q}, j_{3}\right) A\left(\bar{k}, j_{4}\right)\left[\delta_{j_{3}, J_{2}} n_{\alpha}^{j_{1}, j_{4}}(\bar{k})-\sigma_{j_{1}, j_{4}} n_{\alpha}^{j_{3}, j_{2}}(\bar{k}+\bar{q})\right] \\
& +\frac{U}{N} \sum_{\substack{\bar{k}_{1}, \bar{q}_{1}, j \\
j_{1}, J_{1}, j_{j}}}\left[B^{*}\left(\bar{k}+\bar{q}_{1}, j_{2}\right) B^{*}\left(\bar{k}_{1}-\bar{q}, j_{1}^{\prime}\right) B\left(\bar{k}_{1}, j_{2}^{\prime}\right) B\left(\bar{k}+\bar{q}-\bar{q}_{1}, j_{3}^{\prime}\right) \times\right.
\end{aligned}
$$

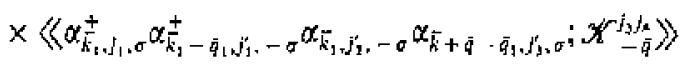

$$
\begin{aligned}
& +B^{*}\left(\bar{k}_{1}+\bar{q}_{1}, j_{1}^{\prime}\right) B^{*}\left(\bar{k}+\bar{q}_{1}, j_{2}\right) B\left(\bar{k}+\bar{q}+\bar{q}_{1}, j_{2}^{2}\right) B\left(\bar{k}_{1}, j_{3}^{\prime}\right) \times
\end{aligned}
$$

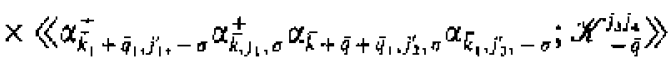

$$
\begin{aligned}
& +B^{*}\left(\bar{k}+\bar{q}_{1}, j_{1}^{\prime}\right) B^{*}\left(\bar{k}-\bar{q}_{1}, j_{2}^{\prime}\right) B\left(\bar{k}_{,} j_{1}\right) B\left(\bar{k}_{1}, \bar{j}_{3}\right) \times
\end{aligned}
$$

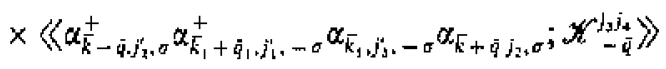

$$
\begin{aligned}
& +B^{*}\left(\bar{k}+\bar{q}, j_{1}^{\prime}\right) B^{*}\left(\bar{k}_{1}-\bar{q}_{1}, \bar{j}_{2}^{\prime}\right) B\left(\bar{k}_{1}, j_{3}^{\prime}\right) B\left(\bar{k}_{j_{1}}\right) \times
\end{aligned}
$$

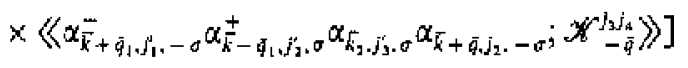

$$
n_{\alpha}^{j_{1}, j_{2}}(\bar{k})=\left\langle\alpha_{\bar{k}_{1}, \sigma_{1}}^{+} \alpha_{\bar{k}_{j_{2}, 0}}\right\rangle
$$

The higher order Green's functions that appear on the right side in (43) are decoupled in the spitit of RPA according to the following scheme:

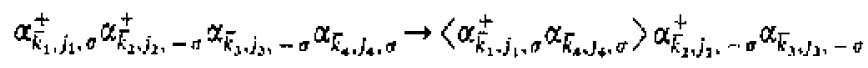

$$
\begin{aligned}
& +\left\langle\alpha_{k_{2}, j_{2},-a}^{+} \alpha_{\bar{k}_{3}, j_{3}, a}\right\rangle \alpha_{k_{1}, j_{1}, \sigma}^{+} \alpha_{\bar{k}_{+}, j_{4}, \sigma}
\end{aligned}
$$

Then (43) reduces to

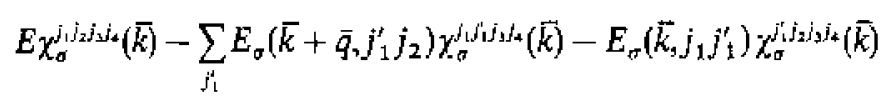

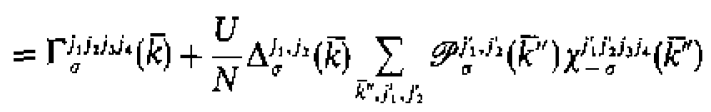


where

$$
\begin{aligned}
& E_{f}\left(\bar{k}, j_{1} j_{2}\right)=E\left(\bar{k}, j_{1}\right)+U n_{f,-a} B^{*}\left(\bar{k}, j_{2}\right) B\left(\bar{k}_{9} j_{1}\right) \\
& \Gamma_{\sigma}^{j_{j} j_{2} j_{3} j_{4}}(\bar{k})=A^{*}\left(\bar{k}+\bar{q}_{1} j_{3}\right) A\left(\overline{k_{3}} j_{4}\right)\left[\delta_{j_{3}, j_{2}} n_{\sigma}^{j_{1}, j_{4}}(\bar{k})-\delta_{j_{1}, j_{4}} n_{\sigma}^{j_{3}, j_{2}}(\vec{k}+\bar{q})\right] \\
& \Delta_{\sigma}^{\prime \prime 2}(\bar{k})=\sum_{j_{3}}\left[B^{*}\left(\bar{k}+\bar{q}, j_{2}\right) B\left(\bar{k}, j_{1}\right) h_{\sigma}^{i_{1}, j_{2}}(\bar{k}+\bar{q})-B^{*}(\bar{k}+\bar{q}) B\left(\bar{k}, j_{2}^{\prime}\right) n_{\sigma}^{j_{\alpha}, j_{2}^{\prime}}(\bar{k})\right] \\
& P^{j_{1} j_{2}}(\bar{k})=B^{*}\left(\bar{k}, j_{1}\right) B\left(\bar{k}+\vec{q}, j_{2}\right)
\end{aligned}
$$

and $n_{f \sigma}$, the average number of $f$-electrons with spin $\sigma$ is given by

$$
n_{f, \sigma}=\frac{1}{N} \sum_{\bar{k}_{J_{1}, j_{2}}} B^{*}\left(\vec{k}_{,} j_{1}\right) B\left(\overrightarrow{k_{,}} j_{2}\right) n_{\sigma}^{j_{1}, j_{2}}(\vec{k})=\left\langle f_{i \sigma}^{+} f_{i \sigma}\right\rangle
$$

We treat pairs of $j$-indices as a single index such that $(11) \rightarrow 1,(12) \rightarrow 2,(21) \rightarrow 3$, and $(22) \rightarrow 4$ so that we can consider

and

$$
\Gamma_{\sigma}^{j_{1} j_{2} j_{3} j_{4}}(\bar{k})=\Gamma_{\sigma}^{\alpha \beta}(\bar{k})
$$

$$
D_{\sigma}^{j_{\sigma} j_{1} j_{1} j_{4}}(\bar{k}, \bar{q}, \bar{E})=D_{a}^{\alpha \beta}(\bar{k})
$$

to be $4 \times 4$ matrices with

$$
\begin{aligned}
& D_{\sigma}^{\sigma g}(\bar{k})= \\
& {\left[\begin{array}{cccc}
{\left[E-E_{\sigma}(\bar{k}+\bar{q}, 11)\right.} & E_{\sigma}(\bar{k}+\bar{q}, 12) & -E_{\sigma}(\bar{k}, 12) & 0 \\
\left.+-E_{\sigma}(\bar{k}, 11)\right] & & & \\
E_{\sigma}(\bar{k}+\bar{q}, 21) & \begin{array}{c}
{\left[E-E_{\sigma}(\bar{k}+\bar{q}, 22)\right.} \\
\left.+E_{\sigma}(\bar{k}, 11)\right]
\end{array} & 0 & -E_{\sigma}(\bar{k}, 12) \\
-E_{\sigma}(\bar{k}, 21) & 0 & {\left[E-E_{\sigma}(\bar{k}+\bar{q}, 11)\right.} & E_{\sigma}(\bar{k}+\bar{q}, 12) \\
& & \left.+E_{\sigma}(\bar{k}, 22)\right] & \\
0 & -E_{\sigma}(\bar{k}, 21) & E_{\sigma}(\bar{k}+\bar{q}, 21) & {\left[E-E_{\sigma}(\bar{k}+\bar{q}, 22)\right.} \\
& & & \left.+E_{\sigma}(\bar{k}, 22)\right]
\end{array}\right]}
\end{aligned}
$$

Further, we treat

$$
\Delta_{\pi}^{j, j}(\bar{k})=\Delta_{\sigma}^{\alpha}(\bar{k})
$$

as a column thatrix and

$$
P^{\lambda_{1 j}(\bar{k})}=P^{\alpha}(\bar{k})
$$

as a row matrix. Then we can rewrite (45) as a matrix equation

$$
\sum_{\bar{\beta}} D_{\sigma}^{\alpha \beta}(\bar{k}) \chi_{\sigma}^{\beta \gamma}(\bar{k})=\Gamma_{\sigma}^{\alpha y}(\bar{k})+\frac{U}{N} \Delta_{\sigma}^{z}(\bar{k}) \sum_{k^{\prime \prime}, \hat{T}} P^{\beta}\left(\bar{k}^{\prime \prime}\right) \chi_{-\sigma}^{\beta y}\left(\overline{k^{\prime \prime}}\right) \text {. }
$$

Muitiplying the above equation from the left by the inverse matrix $D_{\sigma}^{-1^{k x}}(\bar{k})$ and summing over $\alpha$ we get

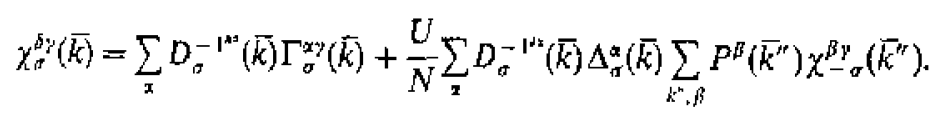


From (56) we see that the expression for $\chi_{\sigma}$ contains $\chi_{-a}$ the origin of which can be traced back to the Hubbard correlation. A little algebra eliminates this and the final expression is given by

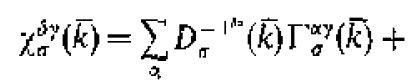

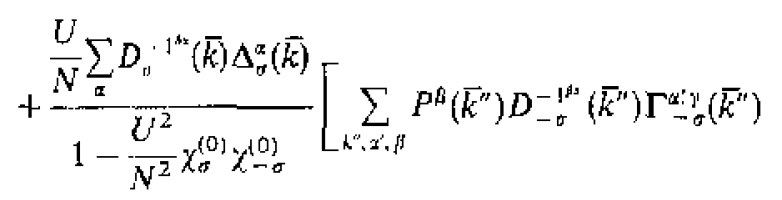

$$
\begin{aligned}
& \left.+\frac{U}{N} \chi_{\sigma}^{(0)} \sum_{k^{\prime \prime}, x^{\prime}, \beta} P^{\mu}\left(\overline{k^{\prime \prime}}\right) D_{\sigma}^{-\Delta^{\prime \prime \prime}}\left(\overline{k^{\prime \prime}}\right) \Gamma_{\sigma}^{\alpha^{\prime} y}\left(\overline{k^{\prime \prime}}\right)\right]
\end{aligned}
$$

where $\chi_{\sigma}^{(0)}$, the $f$-electron susceptibility is given by

$$
\chi_{\sigma}^{(0)}(\bar{q}, E)=\sum_{k, \alpha, \delta} P^{d}(\bar{k}) D_{\sigma}^{-11^{\Delta x}}(\bar{k}) \Delta_{n}^{\alpha}(\bar{k})
$$

Using (57) in (39) compietes the derivation for $\left\langle\rho_{\bar{q}} ; \rho_{-\bar{\pi}}\right\rangle$. The right side of (57) however involves, apart from the model parameters, the ensemble averages $n_{\sigma}^{j j^{\prime}}(\bar{k})$. These quantities can be calculated from the knowledge of single-particle Green's functions. In the next section we sketch briefly the calculation of the required single-particle Green's function and the ensemble averages.

\section{Single-particle Green's functions}

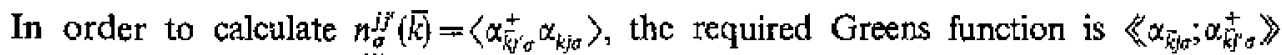
which we denote by $G_{\sigma}^{j j^{\prime}}$.

The equation-of-motion satisfied by $G_{\sigma}^{j{ }^{\prime}}$ is

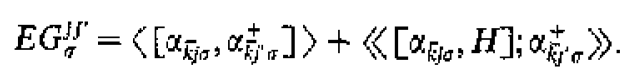

Calculating the commutators on the right hand side this reduces to

$$
\begin{aligned}
& {[E-E(\bar{k}, j)] G_{\sigma}^{j \prime}=\delta_{j, j^{\prime}}+\frac{U}{N} \sum_{\substack{k_{1}, q_{j} \\
j_{1} j_{j}, j}} B^{*}(\bar{k}, j) B^{*}\left(\bar{k}+\bar{q}, j_{1}\right) B\left(\bar{k}, j_{2}\right) B\left(\bar{k}+\bar{q}, j_{3}\right) \times}
\end{aligned}
$$

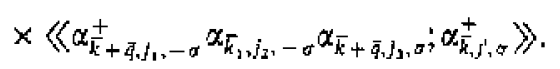

The higher order Green's function occurring on the right side is reduced by resorting to the decoupling

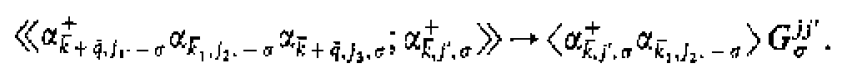

Then all the Green's functions appearing in the equation of motion will be of the same order and we can write

$$
[E-E(\bar{k}, j)] G_{\sigma}^{j j^{\prime}}-U n_{f-\sigma} \sum_{,^{\prime \prime}} B^{*}(\bar{k}, j) B\left(\vec{k}, j^{\prime \prime}\right) G_{\sigma}^{j^{\prime \prime} j^{\prime}}=\dot{j}_{j, f^{\prime}}
$$


A little manipulation then yields the resuit

where

$$
G_{\sigma}^{j j^{\prime}}=\sum_{\zeta^{\prime}}\left(1-\delta_{j j^{\prime}}\right) \frac{\left[E-E_{\sigma}\left(\bar{k}_{, j^{\prime} j^{\prime \prime}}\right)\right] \delta_{j j^{\prime}}+E_{\sigma}\left(\bar{k}_{*} j^{\prime} j\right) \delta_{j^{\prime}}}{\left[E-E_{\alpha}^{+}(\bar{k})\right]\left[E-E_{\sigma}^{-}(\bar{k})\right]}
$$

$$
E_{\sigma}^{ \pm}(\bar{k})=\frac{1}{2}\left[\varepsilon_{\bar{k}}+E_{f}+U n_{f-\pi}\left(\left(\varepsilon_{\bar{k}}-E_{f}-U n_{f-\sigma}\right)^{2}+4 V_{k}^{2}\right)^{1 / 2}\right]
$$

are the poles of the Green's function. The well-known spectral theorem can now be used to evaluate the averages $\left\langle\alpha_{\overline{k j q}}^{+} \alpha_{\overline{k j} a}\right\rangle$ and the results are

$$
\begin{aligned}
& n_{\sigma}^{11}(\bar{k})=\frac{1}{E_{\sigma}^{+}-E_{\sigma}^{-}}=\left[\frac{E_{\sigma}^{-}-E_{\sigma}(\overline{k,} 22)}{1+\exp \left(\beta E_{\sigma}^{+}\right)}-\frac{E_{\sigma}^{-}-E_{\sigma}(\bar{k}, 22)}{1+\exp \left(\overline{\beta E_{\sigma}^{-}}\right)}\right], \\
& n_{\sigma}^{22}(\bar{k})=\frac{1}{E_{\sigma}^{+}-E_{\sigma}^{-}}\left[\frac{E_{\sigma}^{+}-E_{\sigma}(\bar{k}, 11)}{1+\exp \left(\beta E_{\sigma}^{+}\right)}-\frac{E_{\sigma}^{-}-E_{\sigma}(\bar{k}, 11)}{1+\exp \left(\beta E_{\sigma}^{-}\right)}\right], \\
& n_{\sigma}^{12}(\bar{k})=\frac{E_{\sigma}(\bar{k}, 12)}{E_{\sigma}^{+}} \frac{1}{-E_{\sigma}^{-}}\left[\frac{1}{1+\exp \left(\overline{\beta E_{\sigma}^{+}}\right)}-\frac{1}{1+\exp \left(\bar{\beta} E_{\sigma}^{-}\right)}\right], \\
& n_{\sigma}^{21}(\bar{k})=\frac{E_{\sigma}(\bar{k}, 21)}{E_{\sigma}^{+}}\left[\frac{1}{-E_{\sigma}^{-}}\left[\frac{1}{1+\exp \left(\beta E_{\sigma}^{+}\right)}-\frac{1}{1+\exp \left(\beta E_{\sigma}^{-}\right)}\right] .\right.
\end{aligned}
$$

In order to numerically evaluate these quantities, however, one needs to know the average $f$-electron number $n_{f_{\sigma}}$, the average $d$-electron number $n_{d \sigma}=\left\langle d_{\sigma}^{\dagger} d_{\sigma}\right\rangle$ and also the chemical potential $\mu$. These three quantities can be obtained by the self-consistent numerical solution of the following equations.

and

$$
\begin{aligned}
& n_{f \sigma}=\frac{1}{N} \sum_{\bar{k}} \frac{1}{E_{\sigma}^{+}-E_{\sigma}^{-}}\left[\frac{E_{\sigma}^{+}-\varepsilon_{\bar{k}}}{1+\exp \left(\beta E_{\sigma}^{+}\right)}-\frac{E_{\sigma}^{-}-\varepsilon_{\bar{k}}}{1+\exp \left(\beta E_{\sigma}^{-}\right)}\right], \\
& n_{d \sigma}=\frac{1}{N} \sum_{\tilde{h}} \frac{1}{E_{\sigma}^{+}-E_{\sigma}^{-}}\left[\frac{E_{a}^{+}-E_{f}}{1+\exp \left(\beta E_{\sigma}^{+}\right)}-\frac{E_{\sigma}^{-}-\varepsilon_{\bar{b}}}{1+\exp \left(\beta E_{\sigma}^{-}\right)}\right],
\end{aligned}
$$

$$
N=\sum_{\sigma}\left(n_{f \sigma}+n_{d \sigma}\right)=\text { constant }
$$

\section{Summary and conclusions}

The energy and wawevector-dependent dielectric function for the Anderson lattice is studied within the linear response theory. The dielectric function is related to the retarded Green's function of the Fourier components of the density fluctuations. The periodic Anderson Hamiltonian is expressed in terms of hybridized band operators. The retarded Green's function is evaluated using the equation-of-motion method. The higher order Green's functions that appear in the chain of equations are decoupled in the random phasc approximation. The final expression for the retarded two-particle Green's function contains thermodynamic averages which can be calculated only from certain single-particle Green's functions. Therefore these single-particle Green's functions are also calculated using the equation-of-motion method and within RPA. 


\section{Acknowledgements}

The author thanks Dr G Gangadhar Reddy for collaboration.

\section{References}

Auderson P W 1961 Phys. Rev. 12441

Gangadhar Reddy G and Ranakanth A 1990 Physica B162 74

Gangadhar Reddy G and Ramakanth A 1991 Sotid State Commun. 78 I33

Hubbard J 1963 Proc. R. Soc. London A276 238

Jayaraman A, Singh A K, Chatterjee A and Usha Devi S 1974 Phys. Rev, B9 2153

Kubo R 1957 J. Phys. Soc. Jpn 12570

Lee P A, Rice T M, Serene J W, Sham L J and Wilkins J W 1986 Comments Cond. Mat. Phys. 1299

Mahan G D 1981 Many-particle physics (New York: Plenum Press)

Newns D M and Read N 1987 Adv. Phys. 36799

Prabbu R B and Tangsali R B 1988 Phys. Status Solidi B149 623

Riseborough F S and Mills D L 1980 Phys. Rev. 8215338

Spalek J 1988 Phys. Rev. B38 208

Varma C M 1976 Rev. Mod. Phys. 48219

Wohlleben D and Wittershagen B 1985 Adv. Phys. 34403

Zubarev D N 1960 Usp. Fiz. Nauk 7171 